\title{
Metodologías activas, la clave para el cambio de la escuela y su aplicación en épocas de pandemia
}

\section{Active methodologies, the key to changing schools and their application in times of pandemic}

Juan José Rocha Espinoza

(D) https://orcid.org/0000-0001-7886-2194

Universidad Politécnica Salesiana, Ecuador

Autor para correspondencia: jrocha@ups.edu.ec

Fecha de recepción: 26 de agosto de 2020 - Fecha de aceptación: 10 de diciembre de 2020

\section{Resumen}

El presente documento pretende realizar una reflexión sobre la propuesta de las Metodologías Activas del Aprendizaje, basado en el socio-constructivismo. Esta implementación propone un giro en el proceso metodológico de la enseñanza, por ende, el cambio hacia una escuela mucho más amigable, que propone un enfoque formativo hacia la resolución de problemas de la vida cotidiana. Si bien es cierto esta propuesta no es nueva, sin embargo, bajo la situación de confinamiento y la imposición de la educación virtual es crucial su aplicación en los procesos de enseñanza aprendizaje. En este manuscrito fue necesario realizar una contextualización de la realidad educativa ecuatoriana mediante el tiempo de pandemia y confinamiento en los hogares, pues esta situación demandó cambios estratégicos en el ámbito educativo. Es así, que se pretende proponer acciones de transformación metodológica mientras el proceso educativo siga estandarizado en la virtualidad y a posterior de igual manera, pues al momento del cotejo de la información, se tuvo que priorizar aquellos datos de relevancia que aparecen de manera constante, que van desde el estado de los procesos educativos a nivel escolar estatal hasta la universidad tanto pública como privada. Llegando a concluir que quienes están a cargo de la gestión educativa de los diversos centros educativos privados y públicos opten por propuestas metodológicas más amigables.

Palabras claves: metodologías activas; enseñanza; aprendizaje; COVID-19; Ecuador.

\begin{abstract}
This document aims to reflect on the proposal of Active Learning Methodologies, based on socioconstructivism. This implementation proposes a turn in the methodological process of teaching, therefore, the change towards a much more friendly school, which proposes a formative approach towards solving problems in everyday life. Although this proposal is not new, however, under the confinement situation and the imposition of virtual education, its application in the teachinglearning processes is crucial. In this manuscript, it was necessary to contextualize the Ecuadorian educational reality through the time of pandemic and confinement in homes, as this situation demanded strategic changes in the educational field. Thus, it is intended to propose methodological transformation actions while the educational process continues to be standardized in virtuality and
\end{abstract}


later in the same way, because at the time of collation of the information, it was necessary to prioritize those relevant data that appear constantly, which They range from the state of the educational processes at the state school level to both public and private universities. Coming to the conclusion that those who are in charge of the educational management of the various private and public educational centers opt for more friendly methodological proposals.

Keywords: active methodologies; teaching; learning; COVID-19; Ecuador

\section{Introducción}

A raíz de la situación mundial del COVID-19, el planeta se ha visto obligado a la evolución y cambios para adaptarse a nuevas realidades. Si bien es cierto, la cuestión del COVID ha sido trágica para muchas familias del mundo, no se diga todo lo que ha ocasionado y sigue ocasionando en el Ecuador.

Ante la situación de la educación en tiempos de confinamiento, a raíz de la declaración de estado de Pandemia por la Organización Mundial de la Salud (OMS) ${ }^{1}$, la mayoría de los países en el mundo recurrieron a la alternativa de virtualizar la educación. Si bien es cierto ya existen propuestas de educación virtualizada desde hace algún tiempo atrás, es importante conocer que implica esto. Para Fardoun, González Collazos y Yousef (2020) al referirse sobre este tema la califican " (...) como la modalidad en donde la docencia se desarrolla en su totalidad a través de escenarios o entornos digitales, aunque puede existir algún encuentro físico cara a cara entre el alumnado y docentes" (p. 17 - 22)

Sobre la educación virtual en el Ecuador, existe un conjunto de propuestas de carreras en línea vigentes por varias Instituciones de Educación Superior (IES) antes de la emergencia sanitaria. Sin embargo, a raíz de la declaración de estado de pandemia por la OMS, y de la "(...) declaratoria de estado de excepción por calamidad pública en todo el estado ecuatoriano". (Presidencia de la República, 2020, p. 14) de parte del gobierno, las instancias de control de la Educación Superior, en este caso el Consejo de Educación Superior (CES) aprobaron la "Normativa transitoria para el desarrollo de actividades académicas en las Instituciones de Educación Superior, debido al estado de excepción decretado por la emergencia sanitaria ocasionada por la pandemia de COVID-19". (CES, 2020, p. 6) para permitir que todos los procesos de enseñanza-aprendizaje a nivel universitario pueda ser realizado mediante la modalidad en línea.

En el caso de la educación a nivel inicial, básica y bachillerato, el Ministerio de Educación (MiniEduc), no propuso ninguna reforma a la normativa legal, sino mecanismos de adaptación a esta emergencia mediante la propuesta del Currículo Compactado. El escenario en esos niveles educativos que regenta el MiniEduc es el más complejo, pues a raíz de las problemáticas que emergen de la misma como la conectividad, formación docente, uso de TIC's,

${ }^{1}$ Después de hacer un recuento rápido de la propagación del coronavirus COVID-19 por fuera de China, el director de la Organización Mundial de la Salud (OMS), Tedros Adhanom Ghebreyesus, dijo este 11 de marzo que en los próximos días y semanas esperan ver que "el número de casos de COVID-19, el número de muertes y el número de países afectados aumente aún más (...) Por lo tanto, hemos evaluado que el COVID-19 puede caracterizarse como una pandemia". (France24, 2020) 
etc., también proyectan los diversos problemas que están entrelazados con los mencionados con anterioridad, en este sentido se hace referencia a la realidad social, económica e inclusive sanitaria. Se convierte inclusive en un caso sistemático de estudio en la que, si se toma como referencia una sola escuela pública, de pocos estudiantes en un sector específico, se refleja toda una realidad nacional en la que se manifiesta toda la inequidad social que conforma este todo llamado Ecuador. Es como una propuesta hologramática, en la que “(...) no sólo las partes están en el todo, sino que el todo está en el interior de las partes”. (Ballesteros, 2011, p.9) se convierte en un diagnostico basado en una muestra, en la que se refleja una totalidad que, por más de estar seccionada, no deja de proyectar la realidad.

En el mismo nivel de educación que está supervisado por el MiniEduc, no solo existen instituciones educativas públicas, si no está conformado por escuelas y colegios particulares. Esta temática será profundizada en algunos párrafos del presente manuscrito.

\section{Metodología}

Teniendo en cuenta las circunstancias en las que se desenvuelve el entorno mundial hasta el mes de agosto 2020, con una población ecuatoriana aún en confinamiento en su mayoría de ciudades, evitando el contacto físico, priorizando la virtualidad. Esta investigación aprovecha los medios electrónicos para realizar un conjunto de entrevistas a diversas personas que conforman los procesos educativos, entre estos, estudiantes universitarios, madres de familia, docentes de instituciones educativas.

En este sentido, es importante comentar que se basa en la percepción de los antes mencionados, pues son quienes han estado inmersos en estos procesos educativos y han palpado de primera mano el desarrollo de estos mediante la virtualidad. Por ende, mantiene un enfoque cualitativo $^{2}$, utilizando la técnica de la entrevista abierta, recogiendo información de gran valor que se da a partir de las vivencias de cada persona involucrada.

De acuerdo con la información que se adquirió, al momento de procesar la misma se procuró tomar aquellos rasgos más objetivos de las entrevistas para que no quede en una simple percepción casuística, sino que aquellos indicios que se dan de manera constante en cada entrevista sirven de mucho para poder conocer la realidad en la que se está caminando en esta educación en tiempos de pandemia. Esto es importante pues se profundiza varios escenarios a nivel educativo donde se trabaja con sujetos de diversas edades.

\section{Vivencias de la virtualidad}

En el presente apartado, se propone una construcción basada en los resultados de las entrevistas, los cuales dan apertura a poder visualizar los escenarios en los que la educación virtual ha sido implementada a pesar de las diversas circunstancias que ya existían en las distintas realidades educativas.

2 "El enfoque cualitativo - por ejemplo, mediante grupos de conversación, entrevistas, testimonios y en general documentos- es exclusivo del orden social y, por lo mismo, no cabe encontrar antecedentes en las ciencias naturales y sus modelos del saber metódico" (Canales, 2006, p.19)

Esta obra se comparte bajo la licencia Creative Common Atribución-No Comercial 4.0 International (CC BY-NC 4.0) Revista de la Universidad Internacional del Ecuador. URL: https://www.uide.edu.ec/ 
De esta forma, se propone un análisis profundo a partir de las perspectivas de las personas que colaboraron con la información, además, se aporta con fuentes periodísticas en la que se expone la realidad educativa bajo la virtualidad. Por ende, se proponen dos subapartados uno dedicado a la educación escolar y otra a la educación universitaria.

\section{Escenario de etapa escolar}

Sin duda, este es el más complejo y el que más necesita atención de parte de las autoridades. Teniendo en cuenta que según cifras del Ministerio de Educación (2020), el sistema educativo tiene una tasa de matrícula de 4`404.571 estudiantes que la conforman hasta el año 2019, si bien es cierto está en relación con el año pasado, es un buen reflejo de la realidad.

\section{Figura 1}

Estudiantes Matriculados 2018 - 2019

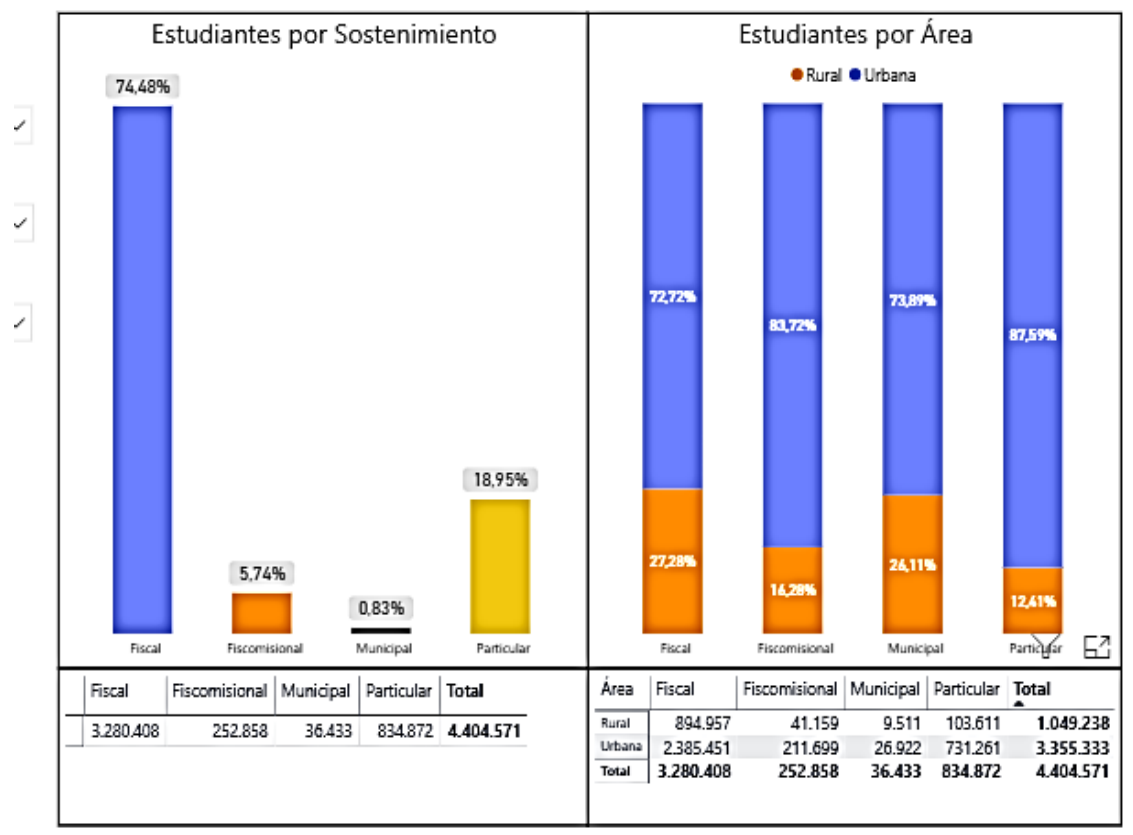

Fuente: Registros administrativos (AMIE). Estudiantes matriculados en instituciones educativas.

Este mismo reflejo, indica que de la cantidad antes mencionada el $74,8 \%$ pertenece a instituciones fiscales, el 5,74 a instituciones fiscomisionales, el 0,83\% a municipales y el 19, 95\% a particulares, a partir de estos datos estadísticos se dará apertura al debate.

Dentro de la estadística habrá una variación, la cuál será analizada más adelante, sin embargo, por ahora la confrontación será con la cantidad de estudiantes que pertenecen al segmento de escuelas y colegios fiscales, se habla de 3’280.408 que, en las condiciones actuales basadas en el confinamiento y la pandemia, está recibiendo las respectivas clases. Sobre este 
tema, Mónica Vargas, madre de familia que decidió cambiar a su hija de una institución privada a una pública, indica que: “(...) las clases que recibe son con apenas dos conexiones vía zoom a la semana, con una duración de 45 minutos, en la que se propone un conjunto de actividades para alcanzar los contenidos del nivel de estudio" (M. Vargas, comunicación personal, 1 de agosto 2020). Esta realidad, no solo es comentada por esta persona, sino es un escenario que se repite a nivel nacional, pues en otra institución de educación fiscal, la docente Violeta Tomalá, indica algo similar: "(...) hacemos reuniones semanales mediante zoom en la que entregamos las respectivas fichas pedagógicas e indicamos a los padres de familia, trabajos a realizar durante la semana, el monitoreo de las tareas y los trabajos se los realiza mediante WhatsApp" (V. Tomalá, comunicación personal, 5 de agosto 2020).

Ante lo mencionado en el párrafo anterior, se pueden generar un conjunto de criterios que comienzan a detallar toda una configuración de la realidad educativa nacional. Pues surgen algunos puntos importantes sobre los cuales reflexionar, entre estos, a) el acceso a la conectividad de estos tres millones de niños y jóvenes que son parte de los procesos educativos gubernamentales, por otro lado, b) la calidad en los procesos educativos, etc.

\section{Acceso a la conectividad}

Este es un punto importante para que se pueda dar el proceso educativo en épocas de confinamiento y pandemia, puesto que el desarrollo de las clases fue propuesto mediante la virtualidad. Para esto el MiniEduc, desde el inicio de la emergencia propuso:

(...) desde el lunes 16 de marzo habilitará una plataforma para que los alumnos puedan estudiar desde casa. Para quienes no tienen facilidades de internet, la ministra de Educación, Monserrat Creamer, dijo que se trabajaría con medios de comunicación de radio y televisión. (El Universo, 2020)

Esta alternativa fue presentada mientras se desarrollaba el ciclo de estudios de la Sierra y Amazonía del país, pues se encontraban en los últimos meses de estudio. Con relación al ciclo Costa, la situación tuvo muchos contratiempos y cuestionamientos, que van desde el aplazamiento del inicio de este que por lo general esta previstos para mediados del mes de abril de cada año, hasta el proceso de virtualización de las clases. Sin embargo, por ahora no se profundizará en estos puntos, sino más bien se pretende hacer énfasis en el acceso a conexión una vez planteada la propuesta del MiniEduc.

Sobre las herramientas que este organismo propuso, existe una especie de plataforma interactiva con recursos digitales ubicado en esta liga https://recursos2.educacion.gob.ec/, en la misma se puede observar un conjunto de recursos distribuidos por año de educación. Esta plataforma pertenece al plan educativo Aprendamos juntos en casa, el cual ha sido preparado por el MiniEduc en respuesta a la emergencia nacional y dar continuidad a los procesos de enseñanza - aprendizaje en el país. Este plan educativo ha diseñado el Currículo Priorizado ${ }^{3}$, o lo que los compañeros docentes reconocen como el currículo compactado.

${ }^{3}(\ldots)$ promueve el desarrollo de habilidades para la vida, como la capacidad de adaptación a la incertidumbre, el desarrollo del pensamiento crítico, el análisis y la argumentación considerando diversas perspectivas, la 
Es necesario tener en cuenta que lo planteado por el MiniEduc, es para todas las instituciones del país, sin embargo, a nivel de educación particular, estas están en libertad de utilizar los mecanismos tecnológicos que crean necesarios. Dentro de las herramientas que se emplean son Moodle, Blackboard, Zoom, Microsoft Team, etc. es interesante por ejemplo que instituciones de particulares de un nivel económico medio emplea el uso de Moodle y Zoom, inclusive con licencias gratuitas, donde la comunicación en una reunión de zoom dura apenas 40 minutos. Por otro lado, las instituciones educativas de un nivel económico alto emplean Blackboard, como es el caso del Sir Thomas Moree, Ecomundo, Delfos, etc.

Una vez expuesta toda la cuestión tecnológica en este nivel educativo, es importante poner en discusión sobre todo al acceso a la tecnología en específico de los estudiantes que pertenecen a instituciones públicas, teniendo en cuenta que:

En Ecuador, solo el 37 por ciento de los hogares tiene acceso a internet, lo que significa que 6 de cada 10 niños no pueden continuar sus estudios a través de plataformas digitales. La situación es más grave para los niños de zonas rurales, solo el 16 por ciento de los hogares tiene este servicio. (Vohlonen, 2020)

A partir de lo que propone la autora, es necesario poder realizar un análisis logrando visualizar si al finalizar el año lectivo 2020-2021 se cumplirán con los objetivos propuestos para que los niños, niñas y jóvenes que conforman el sistema educativo, sobre todo fiscal, logren alcanzar los conocimientos mínimos. En este sentido, Vivanco (2020) haciendo un análisis de la situación educativa virtual a nivel del país expone que, “(...) los hogares del estrato socioeconómico más desfavorecido, aquellos de los grupos tradicionalmente excluidos y marginados están en obvia desventaja al no contar con acceso a internet, lo cual les impide continuar con el proceso educativo de sus hija/os". (p. 5)

En efecto, el acceso a la conectividad de parte de los estudiantes en este caso, padres de familia y estudiantes, desvela una realidad socioeconómica que está entretejida en esta configuración. Ya que no solo se trata de la conectividad, si no de poseer los recursos tecnológicos necesarios para realizar las respectivas conexiones. Como lo indica el diario Expreso "Se calcula que un millón de niños y adolescentes no tienen computador, laptop o un teléfono inteligente; tampoco tienen cuentas de Internet en sus casas ni en sus móviles" (Expreso, 2020). Esta realidad redactada por un diario de la ciudad de Guayaquil, es constatada con la información levantada a raíz de la escritura del presente documento, es así, que Walter Carrasco, docente de la Escuela Fiscal Cerro del Carmen indica que, "el cumplimiento tardío de las tareas y trabajos, e inclusive las no entregas de las mismas, que propone por cada asignatura se debe a que por familia poseen solo un dispositivo móvil, por ende, realizar las tareas cuando se tienen dos o tres hijos se vuelve complicado" (W. Carrasco, comunicación personal, 23 de junio 2020)

Todo lo antes expuesto, lleva a que se cuestione por completo todo el proceso educativo que se está implementando en el país, pues tratando de elaborar un criterio global, predomina un

comunicación empática, la toma de decisiones, el trabajo colaborativo, el manejo de las tecnologías, la formación en valores, la contención emocional de los estudiantes y la participación de las familias. (Ministerio de Educación, 2020) 
panorama desigual para los niños/as y jóvenes del país, pues, si bien es cierto que los padres y madres de familia pueden escoger la educación que deseen para sus hijos/a, como lo indica la Constitución de la República en su artículo $29^{4}$, quienes forman parte del sistema educativo fiscal se encuentran en total desventaja, sobre todo, si pertenecen a espacios rurales.

\section{Calidad en los procesos educativos}

Lo expuesto en el apartado anterior, está relacionado con la calidad en los procesos educativos. Para esto, es necesario tener como punto de partida el plano legal que propone la Constitución de la República, que en su artículo 27, expone que, la educación se centrará en el ser humano y garantizará su desarrollo holístico, en el marco del respeto a los derechos humanos, al medio ambiente sustentable y a la democracia; será participativa, obligatoria, intercultural, democrática, incluyente y diversa, de calidad y calidez; impulsará la equidad de género, la justicia, la solidaridad y la paz; estimulará el sentido crítico, el arte y la cultura física, la iniciativa individual y comunitaria, y el desarrollo de competencias y capacidades para crear y trabajar. (Asamblea Nacional, 2008, p. 33)

Es necesario partir desde lo que dicta la Carta Magna, pues propone muchas características de la educación las cuales deben ser prioridad para el estado ecuatoriano, entre estas, se encuentra la palabra calidad, a la que se hará referencia durante este apartado.

Es cuestionable hablar de una educación de calidad, cuando en el común de los tiempos previos a la pandemia existían muchísimas interrogantes sobre la misma. Ahora, hablar de calidad en la educación en los tiempos de pandemia y confinamiento se convierte en una temática que debe preocupar a todos quienes conforman el sistema educativo. Sobre todo, cuando existen demoras en los pagos salariales de los docentes y estos mismos poseen limitaciones en el uso de la tecnología y de las herramientas virtuales que proponen un aprendizaje activo.

En lo que tiene que ver con la demora en los pagos salariales, el diario el Comercio en una publicación del 18 de agosto del 2020, expone, profesores del sistema público, tanto de educación media como superior, no han recibido su salario correspondiente al mes de julio del 2020. Frente a esta situación, docentes fiscales han empezado a tomar medidas. Este martes 18 de agosto, por ejemplo, se realizó un plantón frente al Ministerio de Educación, ubicado al norte de Quito. (El Comercio, 2020)

Esta situación salarial, no solo comprende un mes de retraso, sino que en los meses anteriores la demora en los pagos llegó hasta tres meses, sobre todo, a nivel de MiniEduc, también esta acarrea el pago de la conexión a internet, la dotación de computadoras en sus hogares. Además, se debe considerar la reducción salarial correspondiente a una hora clase y el despido masivo de docentes en el mes de marzo. Sin duda el sector de la educación recibió el más duro golpe, pues al reducir la cantidad de maestros la carga horaria aumenta para los docentes que se quedan en el sistema educativo.

4 “Las madres y padres o sus representantes tendrán la libertad de escoger para sus hijas e hijos una educación acorde con sus principios, creencias y opciones pedagógicas". (Asamblea Nacional, 2008, p. 33)

Esta obra se comparte bajo la licencia Creative Common Atribución-No Comercial 4.0 International (CC BY-NC 4.0) Revista de la Universidad Internacional del Ecuador. URL: https://www.uide.edu.ec/ 
Por otro lado, la cuestión de la virtualidad conlleva un proceso de capacitación para el manejo de las herramientas tecnológicas, programas y aplicaciones que facilitan la enseñanza aprendizaje en la virtualidad. Este punto no solo afecta a los colegios y escuelas del gobierno, sino a las instituciones particulares, pues es necesario comprender que la situación de confinamiento, pandemia y virtualidad, orilla a la implementación de metodologías activas del aprendizaje (que no solo debe usarse en la virtualidad), al manejo de aplicaciones para implementar la gamificación ${ }^{5}$, etc. Esto no significa que se reduzca la calidad educativa a la simple reproducción de un video durante la reunión de Zoom, como lo indica Verónica Loor, madre de familia, pues comenta, "que tuvo que sacar a su hijo de la institución privada en la que lo tenía, pues era recurrente que la maestra exija conexiones en zoom, reproduzca un video de youtube durante la misma y diga a los padres de familia que realicen esa tarea con los niños/as" (V. Loor, comunicación personal, 8 de agosto 2020)

Es un error pensar que las clases virtuales deben realizarse de manera similar que, en la presencialidad, pasar ocho horas frente a la computadora, mantener una conexión constante con la cámara prendida, llamar la atención porque no está usando el uniforme de la institución, etc. Los procesos educativos no se pueden reducir a la mera propuesta conductista ${ }^{6}$, ni tampoco al facilismo tecnológico de reproducir un video de la actividad en clase, es necesario que se genere contenido de parte del docente, que este prepare su propio material, que investigue sobre el contenido y los diversos métodos para proponer clases activas con trabajos colaborativos ${ }^{7}$ que generen aprendizajes significativos ${ }^{8}$.

Tal vez, el último párrafo sea muy exigente teniendo en cuenta el contexto del pago tardío de los salarios, pero eso no debe disminuir el impulso de construir calidad el cual es generado por la cuestión vocacional de maestros/as. Seguro es difícil hablar de calidad educativa cuando existe una disminución de sueldo y el pago tardío de los mismos, de igual manera cuando el docente posee muchas limitaciones a nivel de conocimiento y empleo de la tecnología.

\section{Escenario a nivel universitario}

A nivel universitario el panorama puede ser un poco variable, por los promedios de edades y el nivel de madurez de los estudiantes, pues se manejan de manera autónoma y cuentan

${ }^{5}$ La gamificación propiamente dicha trata de potenciar procesos de aprendizaje basados en el empleo del juego, en este caso de los videojuegos para el desarrollo de procesos de enseñanza-aprendizaje efectivos, los cuales faciliten la cohesión, integración, la motivación por el contenido, potenciar la creatividad de los individuos. (Marín Díaz, 2015, p. 1)

${ }^{6}$ El conductismo iguala al aprendizaje con los cambios en la conducta observable, bien sea respecto a la forma o a la frecuencia de esas conductas. El aprendizaje se logra cuando se demuestra o se exhibe una respuesta apropiada a continuación de la presentación de un estímulo ambiental específico. (Ertmer \& Newby, 1993)

${ }^{7}$ El trabajo colaborativo promueve en un pequeño equipo de estudiantes a lograr metas comunes, cabe aclarar, que trabajo colaborativo no significa repartir tareas ni es sinónimo de trabajar en grupo, ya que se puede observar que en un trabajo en grupo se puede dar la competencia entre los integrantes o bien la indiferencia y en el trabajo colaborativo la interdependencia positiva entre los miembros del equipo es el factor principal en esta forma de trabajo. (Rosario, 2008, p. 133)

${ }^{8}$ Ausubel (como se citó en Díaz, 2003) durante el aprendizaje significativo el aprendiz relaciona de manera sustancial la nueva información con sus conocimientos y experiencias previas. Se requiere disposición del aprendiz para aprender significativamente e intervención del docente en esa dirección. 
con los recursos tecnológicos para poder llevar este proceso educativo, al menos con un dispositivo móvil para seguir sus clases vía Zoom. Sin embargo, existen algunos puntos importantes a tomar en cuenta como resultado de la virtualidad, como el tiempo sentado frente a la pantalla de la computadora recibiendo clases, el tiempo de realización de tareas; y, la metodología de enseñanza en la virtualidad, esta última incluye el trato de la relación docente estudiante, la capacitación y manejo de herramientas tecnológicas.

Como se expuso en el apartado donde se expone el contexto, la entidad de control CES, realizo el respectivo ajuste a la normativa para poder validar los procesos de enseñanza aprendizaje en la virtualidad. A partir de esta, se brindó las respectivas capacitaciones en las diversas Instituciones de Educación Superior, para el manejo de plataformas digitales. La Universidad Politécnica Salesiana la realizó en el mes de abril, la Universidad de la Artes de igual forma y así cada IES proporcionó los espacios necesarios de formación.

Las plataformas de mayor uso en las IES es Moodle y Zoom, pocas instituciones utilizan otra herramienta como blackboard, de seguro por el costo operativo. En el caso de la Universidad Estatal de Guayaquil, retrasó el inicio de clases al primero de julio, cuando este estaba previsto para el mes de mayo, esta se debió a raíz de la virtualización de la educación y el respectivo soporte a la cantidad de estudiantes. Sin contar con el número de docentes que tuvo que despedir para cubrir el recorte presupuestario propuesto por el gobierno en el mes de mayo 9 .

\section{Procesos educativos en el aula virtual}

La ventaja de proceder con el proceso investigativo en el nivel universitario es que la información que se recoge de parte de los estudiantes posee mayores criterios, manteniendo la objetividad. En este sentido, cuando se les consulta sobre ¿Cómo califican los procesos de implementación de la educación virtual? La respuesta se mantiene en los parámetros de bueno y regular. Esta experiencia es completamente nueva, donde no solo los estudiantes han tenido que adaptarse, sino también los docentes realizan un esfuerzo enorme.

Convertir los procesos educativos presenciales en virtuales, no solo es cuestión de que se determine este cambio mediante la vía legal, sino conlleva que las IES y sus claustros docentes comprendan que el cambio metodológico se encuentra presente en el mismo. Para esto es necesario comprender una definición de lo que se conoce como metodología,

La Metodología es entendida como un concepto global referido al estudio del Método (o de los métodos) desde un proceso sistemático en el cual se adquieren modos y formas de conocimiento; el Método es considerado como el camino para obtener un fin de manera ordenada, desde un conjunto de reglas. (Forero, 2007, p. 123)

Comprender la cuestión metodológica como un procedimiento que comprende formas y pasos para llegar a un fin, en la educación presencial se plantea desde un panorama diferente en la que el espacio, el tiempo, el contacto con la persona se convierten en elementos que construyen todo un escenario diferente a la de la virtualidad, en el que el espacio se convierte en

\footnotetext{
${ }^{9}$ Cfr. (Pichincha Comunicaciones, 2020)
} 
un no lugar, en este sentido no como lo propone del todo Auge (1993) aunque se podría rehacer una lectura de este no lugar, es necesario que el docente contextualice este nuevo escenario e implemente procesos metodológicos para que este espacio sea mucho más activo y menos predecible. Pues, los estudiantes pasan un promedio de 8 horas frente a la pantalla de una computadora para sus clases, sin contar las horas que emplean para realizar las tareas que van de 3 a 6 horas diarias.

Es necesario que se comprenda que el estudio mediante los procesos de virtualidad no puede, ni debe ser igual a los que se desarrolla en la presencialidad. Tal vez exista una desesperación por no perder la calidad educativa, lo cual es comprensible, pero es necesario desaparecer ese paradigma de perder la calidad educativa en la virtualidad, pues bajo una planificación adecuada y manejo de actividades asincrónicas y conexiones no tan extensas vía Zoom, el docente puede monitorear los avances mediante la retroalimentación. En esta vía, Belén Alcívar, estudiante universitaria, expone que "la experiencia de clases virtuales la califica como buena, sin embargo, no es igual estar conectada a una computadora para una clase mediante Zoom, pues por más que el docente realice talleres, la clase se convierten en espacios monótonos donde prácticamente los profesores pasan hablando varias horas como si estuviésemos en presencialidad, es necesario que estos espacios sean más activos" (B. Alcívar, comunicación personal, 1 de agosto 2020)

Esta perspectiva de la estudiante es repetitiva en todos los que fueron consultados, por otro lado, también emergen cuestiones interesantes como la comprensión que el docente debe tener al momento de mantener una conexión en Zoom, pues en el caso de Eduardo Estacio, estudiante universitario, indica que "muchas veces algunos profesores no logran comprender el desafío de la virtualidad y proponen una clase completamente inductiva, donde pretenden obtener los mismo resultados cuando utilizan ese método a nivel presencial". (E. Estacio, comunicación personal, 30 de julio 2020) La virtualidad está propuesta como una modalidad, en este sentido se adopta la misma por la emergencia, sin embargo, la comprensión del contexto general es necesario para poder sobrellevar estos espacios que se plantean como temporales.

Por otro lado, también emerge la cuestión de la capacitación y manejo de la plataforma de parte de los docentes, si bien es cierto las capacitaciones fueron previas al proceso, aún existe una brecha entre la tecnología y el uso de esta de parte de los docentes, lo cual es compresible. Sobre este tema Mercy Cruz, estudiante universitaria, comenta que "al inicio de este proceso educativo era común que algunos profesores no empleen el Moodle para subir los documentos de lectura, sino envíen por WhatsApp, otros al momento de subir los documentos envían una cantidad de lecturas que no tenían conexión con la temática de la clase, e inclusive subían los documentos equivocados de otros ciclos". (M. Cruz, comunicación personal, 8 de agosto 2020)

Es común que, al momento de emplear estos espacios virtuales mediante las plataformas, no solo exista ciertas limitaciones al uso, sino que existe una resistencia paradigmática de reducir sus clases al documento de PDF que se sube, en este sentido, no es una reducción de esta, sino más bien los documentos son insumos para complementar el contenido y su aplicabilidad. 
Se vuelve una tarea necesaria el que no se pierda la centralidad de la educación en el estudiante, el proceso de enseñanza debe de partir desde la cabeza de la persona que aprende, para que el que se enseña pueda adaptar y adaptarse, sobre todo, a estos tiempos y sus desafíos.

\section{Importancia del cambio metodológico}

El presente documento es una construcción que generará muchos cuestionamientos, pues no plantea desde un inicio una línea secuencial con la temática, en este sentido, no se podía dejar de lado la cuestión contextual en la que se desarrolla el análisis, por ende, era necesario partir del contexto para luego exponer la información obtenida y con la misma plantear criterios para mejora de los procesos educativos a nivel del aula, en este caso en la virtualidad.

Más allá de los problemas que se reflejan a nivel social y económico a partir de la situación de la pandemia, deben de ser tomados en cuenta para la toma de decisiones a nivel de estado, en este manuscrito emerge en común la línea metodológica a nivel de la educación propuesta por el MiniEduc y por las diferentes IES. Es así como, se debe comprender que urge la empleabilidad de metodologías para un aprendizaje activo, o de Metodologías Activas del Aprendizaje, que

Corresponden a aquellas metodologías que materializan este cambio en la forma de entender el aprendizaje, ya que se centran en las actividades más que en los contenidos, lo que implica cambios profundos en el actuar de profesores y estudiantes. Esto implica modificar la planificación de las asignaturas, el desarrollo de las actividades formativas y la evaluación de los aprendizajes, de manera tal que se promueva el alineamiento constructivo. (Silva Q. J., y Maturana C. D. 2017, p. 121)

A partir del aporte de los autores, se puede decir que las Metodologías Activas es el conjunto de diversas propuestas de aprendizaje que se basan en actividades que refuerzan los contenidos a aprender y que a partir de estas se construye y refuerza el conocimiento, haciendo que este proceso sea mucho más amigable y se encuentre centrado en la persona que aprende, en este la figura del docente es fundamental pues programa con antelación y acompaña en el mismo.

Si bien es cierto, hablar sobre este tema no causa novedad, pues se puede dar por entendido que en la presencialidad se la aplica, lo cual no es una verdad absoluta. Ya que el método utilizado sigue estando como una propuesta vertical en la que el docente sigue siendo el centro del proceso enseñanza - aprendizaje. Imaginar que el uso de las metodologías activas en la virtualidad no es una idea descabellada, sino desde que se habla de este tema, se van incluyendo las mismas, por ejemplo, incluir plataformas como Moodle, Blackboard; repositorios de audio y video como Youtube, Vimeo, Spotify, etc., es trabajar mediante la propuesta del aula invertida o flipped classroom, en la que inclusive el PDF que se sube a la plataforma y que se propone leer en casa, forma parte de esta. Pues (...) consiste en que el alumno estudie los conceptos teóricos por sí mismo a través de diversas herramientas que el docente pone a su alcance, principalmente vídeos o podcasts grabados por su profesor o por otras personas, y el tiempo de clase se aproveche para resolver dudas relacionadas con el material proporcionado, realizar prácticas y abrir foros de discusión sobre cuestiones controvertidas. (Berenguer, 2016, p. 1466) 
Es necesario no agotar el recurso llamado clases magistrales, pues se convierten en espacios de monologo que muchas veces pueden ser necesarios, pero el aprendizaje plantea muchos desafíos como el de mantener procesos activos, donde este se dé por descubrimiento e investigación, en esta línea se encuentra el Aprendizaje Basado en Proyectos $(A B P)^{10}$ en el que los estudiantes trabajan de manera colaborativa y mediante el desafío de la propuesta investigan y construyen el conocimiento; también el Aprendizaje Basado en Problemas $(A B P r)^{11}$ en el que a partir de una problemática trabajan mediante grupos, investigan y proponen soluciones; además, el Aprendizaje Basado en el Pensamiento (TBL) Thinking-Based Learning ${ }^{12}$, en el que mediante un proceso de investigación profundizan aquello que se da por aceptado, cuestionan, confrontan y crean su propio conocimiento.

Los procesos de enseñanza - aprendizaje se ven enriquecidos mediante estas propuestas metodológicas, lo más importante es que las antes mencionadas, incluyendo gamificación y aprendizaje colaborativo, no se limitan a un nivel específico de educación, lo que quiere decir es que su implementación puede darse desde los inicios de la etapa escolar, hasta la instancia universitaria.

\section{Importancia para la toma de decisiones}

A nivel de gerencia educativa, es necesario conocer las realidades planteadas en este manuscrito, por un lado, a nivel de estado debe de existir una respuesta inmediata que pueda dar solución a las problemáticas encontradas que van desde el acceso a la conexión vía internet, pasando por el pago a docentes (escuelas, colegios institutos técnicos y universidades), sobre todo, proponiendo procesos metodológicos para fortalecer el aprendizaje en este sector. De seguro que ajustando esto detalles neurálgicos, no se verá afectada la calidad educativa.

A nivel de la educación privada en escuelas y colegios, es necesario que se profundice el trabajo metodológico, no es cuestión de replicar las clases presenciales, en las que inclusive el uniforme, el peinado, la cámara encendida se priorizan como si fuesen la centralidad de la educación, es importante humanizar el proceso virtual de tal manera que, a pesar de que exista el distanciamiento social, la experiencia sea muy cercana a las necesidades de los niños/as y jóvenes. Para alcanzar esto, es necesario que desde los rectorados y vicerrectorados se tomen decisiones claras, se adopte una postura personalista con una capacitación a maestros/as para que la escuela en la virtualidad se vuelva amigable con todos los que conforman la Comunidad Educativa.

En lo que corresponde al proceso universitario, a pesar de que existen menos limitaciones como en otros niveles, la cuestión metodológica sigue estando como una constante a sabiendas que, esta comprende un abanico de áreas del conocimiento y que existen espacios de práctica necesarios para la mayoría de asignaturas, quienes tienen la responsabilidad de cada institución deben considerar la empleabilidad de herramientas metodológicas haciendo que el espacio de

${ }^{10}$ Cfr. (Cascales Martínez, A., \& Carrillo-García, M. E. 2018).

${ }^{11}$ Cfr. (Gil-Galván, 2018).

12 Cfr. (Barbán Gari, 2017). 
enseñanza - aprendizaje sea activo, priorice el descubrimiento del conocimiento y la investigación científica. Por otro lado, es fundamental reducir la carga de trabajo del docente, pues bajo esta modalidad es donde más se debe crear contenido, explorar las herramientas para generar una evaluación más abierta mediante la gamificación o mediante el ABP, o alguna otra opción que existe más allá de los simples cuestionarios.

\section{Referencias Bibliográficas}

France24. (2020). OMS: el brote del coronavirus COVID-19 "puede ser caracterizado como una pandemia." Retrieved 13 August 2020, from https://www.france24.com/es/20200311oms-coronavirus-covid19-pandemia-contagios-muertes

Fardoun, H., González-González, C. S., Collazos, C. A., \& Yousef, M. (2020). Estudio exploratorio en Iberoamérica sobre procesos de enseñanza-aprendizaje y propuesta de evaluación en tiempos de pandemia. Education in the Knowledge Society, 21, 17. doi:10.14201/eks.23437

Presidencia de la República. (2020). Decreto Presidencial 1017 (pp. 1-18). Quito.

CES. (2020). Normativa transitoria para el desarrollo de actividades académicas en las Instituciones de Educación Superior, debido al estado de excepción decretado por la emergencia sanitaria ocasionada por la pandemia de COVID-19”. (pp. 1- 6). Quito.

Ballesteros, M. (2011). Pensamiento complejo y estudio de la comunicación. Phonica, 7, 3-18.

Canales, M. (2006). Metodologías de investigación social (1st ed., pp. 1 - 396). Santiago: Lom Ediciones.

Ministerio de Educación. (2020). Visualizador Estadístico - Educar Ecuador | Ministerio de Educación. Retrieved 20 August 2020, from https://educarecuador.gob.ec/visualizadorestadistico/

Ministerio de Educación. (2020). Currículo Priorizado para la Emergencia. Recursos2.educacion.gob.ec . Recuperado 20 de agosto de 2020, a partir de https://recursos2.educacion.gob.ec/wp-content/uploads/2020/08/Curr\%C3\%ADculoPriorizado-para-la-Emergencia-2020-2021-1.pdf

El Universo. (2020). ¿Cómo funciona la plataforma virtual para los estudiantes por la suspensión de clases en Ecuador? El Universo, p. Página Web.

Anna Vohlonen, «COVID-19: Cómo asegurar el aprendizaje de los niños sin acceso a Internet» UNICEF (2020). https://www.unicef.org/ecuador/historias/covid-19-c\%C3\%B3moasegurar-el-aprendizaje-de-los-ni\%C3\%B1os-sin-acceso-internet

Vivanco-Saraguro, A. (2020). Teleducación en tiempos de COVID-19: brechas de desigualdad. CienciAméRica, 9(2), 166-175. doi:10.33210/ca.v9i2.307

Expreso. (2020). Las clases virtuales tomaron a los alumnos 'fuera de línea'.www.expreso.ec. Recuperado 21 de agosto de 2020, a partir de https://www.expreso.ec/guayaquil/clases-virtuales-tomaron-alumnos-fuera-linea12659.html

Asamblea Nacional. (2020). Constitución de la República del Ecuador (págs. 1 - 216). Quito: Asamblea Nacional.

El Comercio. (2020). Profesores fiscales y maestros universitarios no han cobrado su salario del mes de julio hasta este 18 de agosto del 2020. El Comercio. Recuperado 23 de agosto de 
2020, a partir de https://www.elcomercio.com/actualidad/profesores-fiscales-maestrosuniversitarios-sueldos.html

Marín-Díaz, V. (2015). La Gamificación educativa. Una alternativa para la enseñanza creativa. Digital Education Review, (27).

Ertmer, P., \& Newby, T. (1993). Conductismo, cognitivismo y constructivismo: una comparación de los aspectos críticos desde la perspectiva del diseño de instrucción. Performance improvement quarterly, 6(4), 50-72.

Rosario, H. (2008). La web. Herramienta de trabajo colaborativo." Experiencia en la Universidad de Carabobo". Pixel-Bit. Revista de Medios y Educación, (31), 131-139.

Díaz Barriga Arceo, F. (2003). Cognición situada y estrategias para el aprendizaje significativo. Revista electrónica de investigación educativa, 5(2), 1-13.

Pichincha Comunicaciones. (2020). Universidades del Ecuador denuncian el presupuesto presupuestario para sueldos y salarios - Pichincha Universal. Pichincha Comunicaciones EP. Recuperado 24 de agosto de 2020, a partir de http://www.pichinchacomunicaciones.com.ec/universidades-del-ecuador-denuncianrecorte-presupuestario-para-sueldos-y-salarios/

Forero, N. A. G. (2007). Metodología, método y propuestas metodológicas en Trabajo Social. Revista Tendencias \& Retos, (12), 119-135.

Augé, M. (1993). Los no lugares: espacios del anonimato. Barcelona: Gedisa.

Silva Q. J., \& Maturana C. D. (2017). Una propuesta de modelo para introducir metodologías activas en educación superior. Innovación educativa (México, DF), 17(73), 117-131. Recuperado en 25 de agosto de 2020, de http://www.scielo.org.mx/scielo.php?script=sci_arttext\&pid=S1665$26732017000100117 \& \operatorname{lng}=$ es\&tlng=es.

Berenguer Albaladejo, C. (2016). Acerca de la utilidad del aula invertida o flipped classroom. XIV Jornadas de Redes de Investigación En Docencia Universitaria. Universidad de Alicante, 1466-1480. Retrieved from http://rua.ua.es/dspace/handle/10045/59358

Cascales Martínez, A., \& Carrillo-García, M. E. (2018). Aprendizaje basado en proyectos en educación infantil: cambio pedagógico y social. Revista Iberoamericana De Educación, 76, 79-98. https://doi.org/10.35362/rie7602861

Gil-Galván, Rosario. (2018). El uso del aprendizaje basado en problemas en la enseñanza universitaria. Análisis de las competencias adquiridas y su impacto. Revista mexicana de investigación educativa,23(76), 73-93. Recuperado en 25 de agosto de 2020, de http://www.scielo.org.mx/scielo.php?script=sci_arttext\&pid=S1405$66662018000100073 \& \operatorname{lng}=\mathrm{es} \& \ln g=\mathrm{es}$.

Barbán Gari, V. (2017). Efectos del aprendizaje basado en el pensamiento (TBL) en la enseñanza de las ciencias naturales. Implicaciones para la formación del profesorado. 